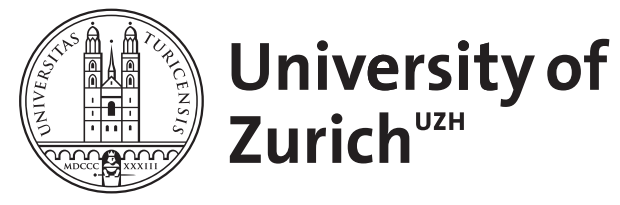

\title{
Replik auf den Leserbrief von Daniel Meili und Susan Dober: Stellungnahme
} der Autoren

\author{
Nordt, Carlos ; Stohler, Rudolf
}

DOI: https://doi.org/10.1007/s00038-004-4111-x

Posted at the Zurich Open Repository and Archive, University of Zurich ZORA URL: https://doi.org/10.5167/uzh-155777

Journal Article

Published Version

Originally published at:

Nordt, Carlos; Stohler, Rudolf (2005). Replik auf den Leserbrief von Daniel Meili und Susan Dober: Stellungnahme der Autoren. Sozial- und Präventivmedizin, 50(1):10-11.

DOI: https://doi.org/10.1007/s00038-004-4111-x 
Psychiatrische Universitätsklinik Zürich, Sektor West und Zentrale Sozialpsychiatrische

Dienste, Zürich

\section{Replik auf den Leserbrief von Daniel Meili und Susan Dober: Stellungnahme der Autoren}

Es freut uns selbstverständlich, dass wir mit unserer Arbeit eine Diskussion ausgelöst haben. Wir stimmen Meili und Dober (2005) insoweit zu, als die Retentionsrate nicht als einziges Effektivitätskriterium von Methadonbehandlungen angesehen werden sollte. Das haben wir aber auch nicht behauptet. Wir halten hingegen daran fest, dass positive Entwicklungen sowie die Vermeidung negativer Folgeerscheinungen meist nur durch eine längere Behandlungsdauer zu erreichen sind.

Als methodische Mängel und Ungenauigkeiten bezeichnen Meili und Dober, dass relevante - wenngleich kaum zuverlässig durch die Leistungsanbieter erfassbare - Variablen (etwa die Komorbidität) nicht in die statistische Analyse einbezogen wurden.

Eine solche Kritik erscheint uns nicht berechtigt, insofern als die zu berücksichtigenden Einflussfaktoren einzig auf Vermutungen gründen. Zentrale Argumente von Meili und Dober basieren auf Annahmen, welche sie durch ihre klinische Erfahrung gewonnen haben. Diese Hypothesen soweit anhand unseres Methadonbehandlungsregisters überprüfbar - sind aber empirisch nicht haltbar. So stellen die beiden Autoren die These auf, dass sich viele Patienten erst nach mehrmaligen Anläufen auf eine längerfristige Behandlung einliessen. Unsere Daten ergeben jedoch keine Hinweise darauf, dass die Behandlungsdauer von Personen mit bereits mehrfach durchlaufenen Substitutionsbehandlungen zunimmt. Dagegen zeigt sich aber eine Verkürzung der Dauer zwischen den einander folgenden Behandlungsepisoden (Nordt et al. 2004).

Auch wird bemängelt, dass nicht auf „konzeptionell intendierte Unterschiede“ zwischen den verschiedenen Leistungsanbietern eingegangen wird. Meili und Dober schreiben mehrfach, dass die Institutionen im Sinne niederschwelliger Zugangsmöglichkeiten funktionierten. Diese Vermu- tung liesse sich belegen, wenn bei den Institutionen mehr Ersteintritte erfolgten als bei Niedergelassenen. Dies ist aber wiederum nicht der Fall. So ist im Zeitraum von 1997 bis 1999 der Anteil der Ersteintritte bei Institutionen $(29,3 \%)$ nicht relevant höher als bei Niedergelassenen $(25,4 \%)$; in absoluten Zahlen ist diese Differenz unerheblich (703 versus 716). Auch die Hypothese, dass sich die Personengruppen je nach Typus der Leistungsanbieter erheblich unterschieden, was die Retentionsunterschiede besser erkläre, kann auf Grund der Registerdaten nicht bestätigt werden. In Tabelle 1 unseres Artikels wiesen wir mit der Darstellung der Unterschiede hinsichtlich Alter, Kokainbeikonsum, Erwerbstätigkeit und Freunden (ausserhalb der Szene) zwischen den beiden Gruppen darauf hin, dass diese nur schwach ausgeprägt sind (Stohler et al. 2004). Selbstverständlich können wir damit aber keine Aussage über möglicherweise relevante Unterschiede in der Motivation, im Vorliegen komorbider Störungen usw. machen (worauf schon im Original-Artikel hingewiesen wurde).

Die Behandlungsdauer in Institutionen könnte nach Meili und Dober auch deshalb kürzer sein, weil diese ihre Patienten nach der festgelegten Frist von 45 Tagen immer rückwirkend auf den letzten Bezugstag abmelden, niedergelassene Ärzte mit Methadonabgabe über die Apotheke ein Behandlungsende aber bis zu drei Monaten zu spät feststellten und (zu spät) meldeten. Uns ist jedoch nur die Vorschrift bekannt, dass Anmeldungen innerhalb 72 Stunden und die Abmeldung unverzüglich zu erfolgen haben. Falls die substituierte Person unabgesprochen kein Methadon mehr bezieht, ist spätestens nach vier Wochen (also 28 Tagen) die Behandlung als beendet zu betrachten (siehe Richtlinien zur methadonunterstützten Behandlung Heroinabhängiger des Kantons Zürich vom 20. März 1996). Träfe die Behauptung von Meili und Dober zu, so würden gravierende Verstösse 
gegen die Methadonabgabe-Richtlinien bestehen. Da aber ein zentrales Ziel der Meldepflicht darin besteht, eine Mehrfachabgabe mit möglicherweise ernsten Konsequenzen zu vermeiden, wäre ein solches Verhalten äusserst bedenklich. Uns liegen allerdings auch hier keine Hinweise für eine massive Häufung solcher Verstösse vor. (Da innerhalb weniger Monate viele Substituierte in eine weitere Methadonbehandlung eintreten, würden diese Doppelbehandlungen bei der Gesundheitsdirektion bemerkt werden, was entsprechend häufige Mahnungen zur Folge hätte).

Das Postulat von Meili und Dober, Langzeitverläufe statt einzelner Behandlungsepisoden zu analysieren, ist prinzipiell sinnvoll. Jedoch ist ihr Lösungsvorschlag - Behandlungen, welche weniger als ein halbes Jahr auseinander liegen, als eine einzige Behandlungsepisode aufzufassen - zu undifferenziert. Es sollte bedacht werden, dass rund ein Drittel der Substituierten bei einem anderen Leistungsanbieter (typus) die folgende Behandlung durchlaufen (34\% wechseln von Institutionen zu Niedergelassenen und 30\% von Niedergelassenen zu Institutionen).

Aufgrund des beschränkten Umfanges einer Replik kann nicht auf jeden Kritikpunkt von Meili und Dober eingegangen werden. Immerhin möchten wir bemerken, dass die Autoren trotz aller Kritik unseren Schlussfolgerungen zustimmen. Unsererseits war es keinesfalls intendiert zu fordern, dass institutionelle Leistungsanbieter von Methadonbehandlungen durch Niedergelassene ersetzt werden sollten. Wir sind davon überzeugt, dass beide Behandlungssettings ihre spezifischen Qualitäten aufweisen. Dies sollte aber nicht verhindern darüber nachzudenken, was von den jeweiligen Leistungsanbietern verbessert werden könnte.

\section{Literaturverzeichnis}

Meili D, Dober $S$ (2005). Replik auf den Artikel „Wie effektiv sind Methadonbehandlungen in Privatpraxen?" von R. Stohler et al. in SPM 49 (2004): 276-80: Leserbrief. Soz Praventiv Med 50: 7-9.
Nordt C, Burri P, Stohler R, Rössler W (2004). Behandlungsunterbrüche mindern die Effektivität von Methadonbehandlungen. Suchtmed 6 : $128-9$.

Stohler R, Nordt N, Falcato L, Dürsteler-MacFarlanc KM, Rössler W (2004). Wie effektiv sind Methadonbehandlungen in Privatpraxen? Soz Praventiv Med 49: 276-80. 